\title{
Electrochemical routes for biomass conversion
}

\author{
Elizabeth J. Biddinger ${ }^{1}\left(\mathbb{D} \cdot\right.$ Oliver Y. Gutierrez $^{2} \cdot$ Jamie Holladay $^{2}$
}

Received: 15 December 2020 / Accepted: 18 December 2020 / Published online: 6 January 2021

(c) The Author(s), under exclusive licence to Springer Nature B.V. part of Springer Nature 2021

There is an urgent need to replace fossil feedstocks currently used in energy and chemical production with renewable carbon resources and energy production to counter the negative effects of climate change and avoid depletion of fossil resources. This transition also will mitigate the environmental impact of the chemical industry. Biomass is an abundant and chemically similar alternative to fossil feedstocks. Use of biomass in chemical and fuel production creates a path toward sustainability when energy from renewable resources also is used. The increasing global capacity to produce energy from renewable resources and the associated decrease in generation costs, with some projections showing the cost of renewable electricity to be less than $\$ 30 / \mathrm{MWh}$ in the United States, could make it economically viable to integrate waste- and biomass-carbon sources with electricity generated from renewable resources. Hence, the natural path forward is to use electrochemistry powered by renewable electricity to drive chemical production.

Electrochemistry offers the possibility of moving beyond simply powering processes with renewable electricity. Its use can provide a foundation for a new approach to chemical processing arising from the (1) the direct interaction of organic molecules with electric fields, (2) the transfer of electrons between surfaces and molecules, (3) the use of external potential as an additional level of control for chemical reactions, (4) controlled specificity of the specific substrates that will be reacted, (5) the ability to operate in dilute solutions that are typical of many waste streams that contain high levels of organic materials, and (6) the absence of external chemical redox species.

Thus, electrochemistry is an interesting and elegant solution for many practical applications involving conversion of

Elizabeth J. Biddinger

ebiddinger@ccny.cuny.edu

1 Department of Chemical Engineering, The City College of New York, CUNY, New York, NY, USA

2 Institute for Integrated Catalysis, Pacific Northwest National Laboratory, Richland, WA, USA biogenic feedstocks, ranging from chemical synthesis and hydrogen generation to waste cleanup. Consequently, there is a renewed interest in identifying opportunities and addressing existing challenges to realize our vision of expanding the use of electrical energy in chemical processes.

Critical challenges to advancing electrochemical conversion of biomass are (1) understanding reaction mechanisms and deriving general kinetic parameters for the conversion of oxygenated molecules; (2) developing electrodes that are active and robust in the presence of aqueous phases and organic compounds; (3) understanding how reaction conditions could be used to control reaction rates, selectivity, and efficiency; (4) designing reactors to optimize electrochemical processes for biomass treatment; and (5) evaluating the economic implications, technical requirements, and life-cycle potential to make electrochemical technologies feasible for different biogenic feedstocks. This special issue showcases current efforts of the electrochemistry community to address these challenges, understand electrocatalysis for biomass conversion, and expand electrochemistry in different directions ranging from selective and controlled reductions and oxidations, to chemical synthesis and treatment of complex mixtures, to valorization and cleaning of waste streams. Our authors aim to understand electrochemical conversion of biomass in increasingly complex environments, design better electrodes, compare electrochemical approaches to traditional thermocatalytic processes, and subject electrochemical processes to the uncertainty of real biomass-derived feedstocks.

The papers in this issue highlight the advantages of using electrochemistry to convert biomass-derived compounds. The studies focused on reductive conversions reflect the kinds of organic compounds that are reactive enough for the reactions to occur at low temperature (e.g., aldehydes, phenolic compounds, and aryl ethers) and highlight the high selectivity associated to electrochemical processes [1-3]. The results highlight the high sensitivity of electrocatalytic processes to reaction conditions (such as $\mathrm{pH}$ and electrolyte), in contrast to many of the reaction condition effects being absent or lessened in thermocatalytic processes [4]. 
Interestingly, these studies report synergies between substrates and electrolytes or among substrates that contradict traditional intuition such as the notion that competitive adsorption decreases reaction rates. For example, these papers provide examples of systems in which electrochemical hydrogenation can provide practical advantages over thermocatalytic hydrogenation [5].

Electrochemical oxidation is shown to be a powerful for functionalizing organic molecules, breaking down macromolecules, and deactivating biologic pathogens [6-8]. This is possible because the applied potential enables oxidative and radical mechanisms in water that otherwise would require the addition of stoichiometric amounts of strong oxidants, which could increase the cost, the chemical hazards and waste generated, and the resulting temperatures in the process. The electrocatalysts used in these oxidations often show deactivation with time, thereby limiting their applications. Hence, advances in the fabrication of robust electrodes and/or development of in situ processes to recover activity are urgent needs along with better reactor and process engineering to decrease the severity of the potential applied while maintaining the desired reactivity [9-11].

The studies published in this special issue use a variety of experimental setups ranging from batch experiments to $\mathrm{H}$-cell processing to slurry and continuous reactors. This variety of approaches shows that the expertise and foundational basis exist for evaluating the requirements needed to scale up processes beyond the laboratory bench. Needed work also includes identifying suitable electrodes, which are also addressed in this special issue. Articles in this issue describe electrodes made of carbon-supported platinumgroup metals, bulk-coinage metals, multi-metallic formulations, metal oxides, and even molecular catalysts that mediate charge transfer between surfaces and substrates. The important message is that it seems that there will not be one, best catalyst for all systems. Electrodes, and their reaction media, will likely need to be adjusted to achieve the desired conversion and selectivity. Having multiple electrode materials and electrolytes, of course, multiplies the complexity of the phenomena under study, thereby creating a steeper learning curve for advancing the related technologies. On the other hand, being able to tune the process by tailoring electrodes and electrolyte compositions also shows the versatility inherent in electrochemistry and provides extensive opportunities for discovery and development in this area.

Complexity, on the other hand, is not a real hurdle to tackling real-world problems. Papers in this issue show that by using electrochemistry, "dirty," ill-characterized mixtures of organic compounds in water can be converted under mild process conditions. Clear advantages of the electrochemical approach mainly are eliminating the need additional chemicals and the possibility of coupling water electrolysis (to form hydrogen as a valuable co-product) with the treatment of organic pollutants and then converting organic pollutants into value-added hydrocarbons. More studies are needed to evaluate the capital costs and power demands of producing widely deployable technologies. However, as with all renewable emerging technologies, there are niche applications that may be suitable for introducing electrochemistry into chemical processing. Finally, energy policies and sociopolitical priorities are steadily shifting toward sustainable industries. Electrochemical conversion of biomass is well suited to be at the forefront of the shift to sustainable processes.

Overall, we present a collection of papers that nicely illustrate the challenges of advancing electrochemical routes for biomass conversion and the efforts of the scientific community to solve those challenges by building on elements of kinetics and catalysis, materials science, organic chemistry, and engineering.

\section{References}

1. Akinola J, Singh N (2020) Temperature dependence of aqueousphase phenol adsorption on Pt and Rh. J Appl Electrochem. https://doi.org/10.1007/s10800-020-01503-3

2. Bababrik R, Santhanaraj D, Resasco DE, Wang B (2020) A comparative study of thermal- and electrocatalytic conversion of furfural: methylfuran as a primary and major product. J Appl Electrochem. https://doi.org/10.1007/s10800-020-01427-y

3. Barton ZJ, Garrett GH, Kurtyka N, Spivey TD, Schaidle JA, Holewinski A (2020) Electrochemical reduction selectivity of crotonaldehyde on copper. J Appl Electrochem. https://doi. org/10.1007/s10800-020-01415-2

4. Wijaya YP, Smith KJ, Kim CS, Gyenge EL (2020) Synergistic effects between electrocatalyst and electrolyte in the electrocatalytic reduction of lignin model compounds in a stirred slurry reactor. J Appl Electrochem. https://doi.org/10.1007/s1080 0-020-01429-w

5. Sanyal U, Koh K, Meyer LC, Karkamkar A, Gutiérrez OY (2020) Simultaneous electrocatalytic hydrogenation of aldehydes and phenol over carbon-supported metals. J Appl Electrochem. https://doi.org/10.1007/s10800-020-01464-7

6. Servedio LT, Lawton JS, Zawodzinski TA (2020) An electrochemical study of cobalt-salen (N,N'-bis(salicylidene)ethylenediaminocobalt)(II) in the oxidation of syringyl alcohol in acetonitrile. J Appl Electrochem. https://doi.org/10.1007/s1080 0-020-01459-4

7. Jafari M, Botte GG (2020) Electrochemical treatment of sewage sludge and pathogen inactivation. J Appl Electrochem. https:// doi.org/10.1007/s10800-020-01481-6

8. Fukushima T, Yamauchi M (2020) Electrosynthesis of glycine from bio-derivable oxalic acid. J Appl Electrochem. https://doi. org/10.1007/s10800-020-01428-x

9. Zhou Y, Shen Y (2020) Electro-oxidation of glycerol by tetrametallic platinum-gold-palladium-silver nanoparticles. J Appl Electrochem. https://doi.org/10.1007/s10800-020-01426-z

10. Bateni F, Ghahremani R, Staser JA (2020) Electrochemical oxidative valorization of lignin by the nanostructured $\mathrm{PbO}_{2} /$ MWNTs electrocatalyst in a low-energy depolymerization 
process. J Appl Electrochem. https://doi.org/10.1007/s1080 0-020-01451-y

11. Lopez-Ruiz JA, Qiu Y, Andrews E, Gutiérrez OY, Holladay JD (2020) Electrocatalytic valorization into $\mathrm{H}_{2}$ and hydrocarbons of an aqueous stream derived from hydrothermal liquefaction. J Appl Electrochem. https://doi.org/10.1007/s10800-020-01452 $-\mathrm{X}$
Publisher's Note Springer Nature remains neutral with regard to jurisdictional claims in published maps and institutional affiliations. 